\title{
Interplay between magnetism and superconductivity in metallic hydrogen and hydrides at high pressure
}

\author{
Lev Mazov ${ }^{1, *}$ \\ ${ }^{1}$ Dept.of Phys.of Supercond., Inst.for Phys.of Microstruct. Russian Academy of Sciences, 603600 Nizhny Novgorod, Russia
}

\begin{abstract}
The detailed analysis of resistive and magnetic measurements with sulfur hydrides at high pressure is performed. The hydrogen system at high pressure can exhibit ferromagnetic (FM) as well as superconducting (SC) properties with layered structure. The onset temperature of resistive transition at $200 \mathrm{~K}$ in metallic sulfur hydrides corresponds to magnetic (AF SDW) phase transition rather than SC one. SC transition in these sulfur hydrides occurs only when magnetic (AF SDW) phase transition is over $(\sim 40 \mathrm{~K})$. The SC mechanism in metallic sulfur hydrides is not conventional but corresponds to Keldysh-Kopaev model characteristic for systems with coexistence of dielectric and SC pairing.
\end{abstract}

\section{Introduction}

According to modern viewpoint, hydrogen at high pressure must pass from the molecular to the metallic phase, which can be SC at relatively high temperatures [1]. However, the experimental implementation of such a transition in laboratory conditions still encounters a number of problems related to the stability of this state. On the other hand, as is known, such pressures are achievable for a number of stars and planets, a significant part of whose composition is hydrogen. Moreover, such celestial bodies, as follows from observational data, have a significant magnetic field. In particular, this is indicated by the polarization of the radio emission observed from "white dwarfs" (WD) [2]. Among the planets in our solar system, as is known, Jupiter and Saturn have the largest magnetic fields, having a large share of hydrogen in their structure (gas giants) (see, e.g. [3]). A recent comparison of theoretical and observational data for magnetic WD indicates that large magnetic field of astrophysical objects is due to monoatomic hydrogen in metallic, FM-ordered state [4].

\section{Hydrogen phase change with increasing pressure}

\subsection{Molecular and metallic hydrogen}

As known, liquid-metal hydrogen in laboratory was obtained under dynamic compression, at a certain density, $\mathrm{P}=140 \mathrm{GPa}$ and $\mathrm{T} \sim 2600 \mathrm{~K}$ (see, e.g. [5,6]). These states were obtained repeating shock compression generated by a shock wave in liquid $\mathrm{H}_{2}$ at $\mathrm{T}=20 \mathrm{~K}$, enclosed between two sapphire anvils. Multiple shock pulse was induced by impact on the cryogenic sample holder by means of either an $\mathrm{Al}$ or $\mathrm{Cu}$ plate accelerated to a certain speed with two-stage light-and-gas gun. The pressures generated were in the range of 90-180 GPa.

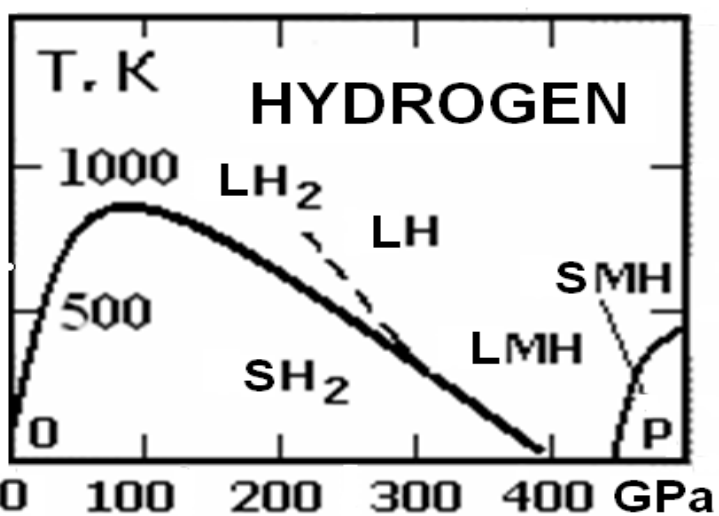

Fig. 1. Phase T-P diagram of hydrogen (see, e.g. [5,6]). (Here, symbols are: $\mathrm{S}$ - solid, $\mathrm{L}$ - liquid, $\mathrm{M}$ - metallic, $\mathrm{H}$ - hydrogen)

The electrical conductivity increased by almost four orders of magnitude between 90 and $140 \mathrm{GPa}$, in which range liquid $\mathrm{H}$ at $\mathrm{T}>1000 \mathrm{~K}$ is a semiconductor with conduction electrons thermally excited through the energy gap. It closes with a density of up to $\sim \mathrm{k}_{\mathrm{B}} \mathrm{T}$ by $\sim$ $0.64 \mathrm{~mol} / \mathrm{cm}^{3}$. At $140 \mathrm{GPa}$ to $180 \mathrm{GPa}$ thermal excitation is quenched and liquid $\mathrm{H}$ is a bad metal $[5,6]$. Measured conductivity $2000 \mathrm{Ohm}^{-1} \mathrm{~cm}^{-1}$ characteristic for liquid metals and highly disordered alloys is in good agreement with the theoretical calculations (see, e.g. $[5,6]$ ).

It is interesting that later, at a static pressure $\mathrm{P}>220$ $\mathrm{GPa}$, a layer-like lattice, called Phase IV, was detected.

Corresponding author: mazov@ipm.sci-nnov.ru 
Phase IV is insulating and, possibly, semi-metallic up to $\mathrm{P} \sim 360 \mathrm{GPa}$, above which, as predicted, it can become metallic (see, e.g. [5,6]). Since the layer-like fcc phase of $\mathrm{H}_{2}$ is similar to graphite in terms of "very low electron charge density in the ground state," Ashcroft [1,7] considered the optical properties of graphite to obtain an understanding of the nature of solid $\mathrm{H}_{2}$ at very high pressures. It was concluded that solid semimetallic $\mathrm{H}_{2}$ at high pressures probably has a layered, crystalline structure of the fcc type and that the paired-H zone in this structure will support this semimetallic phase at very high pressures, thereby preventing a transition to metallic phase at a pressure higher than the pressure in the lamellar phase.

\subsection{Ferromagnetism in metallic hydrogen}

There were put forward two concepts indicating that metallic hydrogen can be FM in nature at high pressure $[3,4]$. The nature of magnetic transition from a paramagnetic (PM) to a FM phase is in fact in a competition between the spin-orientation dependent exchange processes which favor a spin-aligned FM state and the contributions of kinetic energy which favor a PM state [4]. In the ground state a FM solid is formed due to the predominance of exchange effects between parallel spins. In one of above approaches [4], the phase diagram for dense metallic hydrogen can be essentially characterized by two dimensionless parameters

$$
r_{s}=m e^{2} a / \hbar^{2} \text { and } \theta=2 m k_{B} T / \hbar^{2}\left(3 \pi^{2} n\right)^{2 / 3},
$$

corresponding to density and temperature, respectively. Here $m, n$, and $k_{B}$ refer to the mass of a fermion, the number density of the fermions, and the Boltzmann constant, respectively; $a=(4 \pi n / 3)^{-1 / 3}$.

In a FM state the Fermi liquid then induces a magnetic field with the strength $B_{M}=4 \pi \mu_{M} n$, where $\mu_{M}$ denotes the magnetic moment of a fermion, and for an electron, $\mu_{M}=9.28477 \times 10^{-21}$ (erg / G).

The free energy for the strongly coupled $\left(r_{s}>20\right.$ and $\Gamma>10)$ PM electron liquids can been expressed in a parametrized form as [4]

$$
f_{P M}\left(r_{s}, \theta\right)=f_{0}(\theta)+f_{1}(\theta, \Gamma, \Theta),
$$

where $\Gamma=e^{2} / a k_{B} T$. First term $f_{0}(\theta)$ corresponds to the Helmholtz free energy per particle of the ideal-gas electrons in the PM state in units of the thermal energy. The ideal-gas contribution to the free energy is expressed as a balance between those of the chemical potential and of the pressure as

$$
f_{o}(\theta)=\alpha_{0}(\theta)-p_{0}(\theta)
$$

The free-energy formulas for the strongly coupled FM electron fluids in the finite temperature regime, were constructed analogously to the way Eq. (1), where parameter $\theta$ should be then naturally replaced to

$$
\theta_{F}=2 m_{e} k_{B} T / \hbar^{2}\left(6 \pi^{2} n\right)^{2 / 3}[4] .
$$

The resulting magnetic phase diagram for metallic hydrogen was constructed in [4] (see, Fig.2). In this diagram, PM and FM phases correspond to the PM and FM fluids, respectively; S-phase corresponds to bcc crystalline solid. Points $\mathrm{T}_{\mathrm{spf}}$ and $\mathrm{C}_{\mathrm{mag}}$ correspond to the trinle noint and the aritisal nnint for the FMA trancition

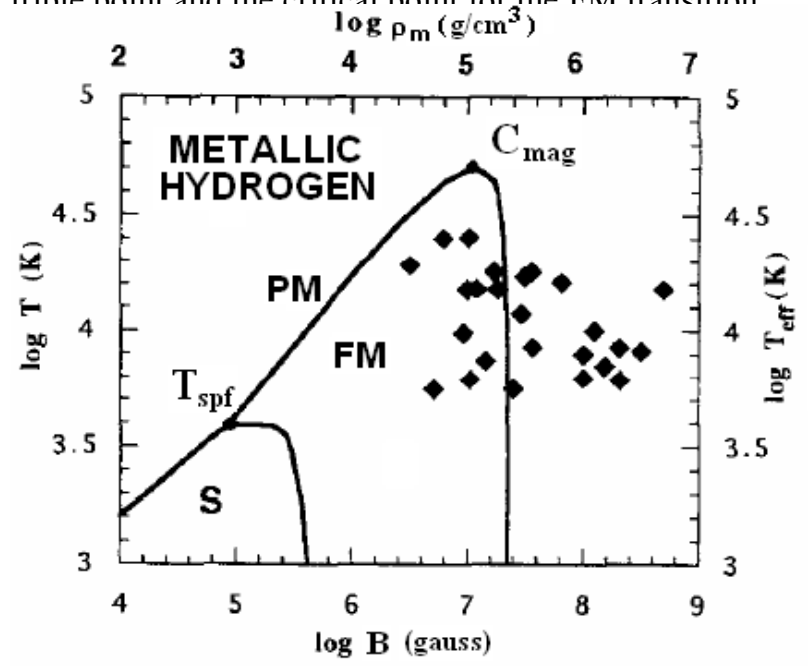

Fig. 2. Magnetic phase H-T diagram of metallic hydrogen [4]. The diamonds represent the data for magnetic white dwarfs estimated from observational data [4] (for legend, see text).

Table 1. Physical parameters at the triple point $\mathrm{T}_{\mathrm{spf}}$ and the critical point $C_{\text {mag }}$ for the metallic hydrogen $\left(\rho_{\mathrm{m}}=m n\right.$ is the mass density)

\begin{tabular}{|c|c|c}
\hline-- & $\mathrm{T}_{\mathrm{spf}}$ & $\mathrm{C}_{\text {mag }}$ \\
\hline$n\left(\mathrm{~cm}^{-3}\right)$ & $3.2 \times 10^{17}$ & $6.0 \times 10^{28}$ \\
\hline$\rho_{m}\left(\mathrm{~g} / \mathrm{cm}^{3}\right)$ & $8.3 \times 10^{2}$ & $1.0 \times 10^{-5}$ \\
\hline $\mathrm{B}_{\mathrm{M}}(\mathrm{G})$ & $8.9 \times 10^{4}$ & $1.1 \times 10^{7}$ \\
\hline$r_{\mathrm{s}}, R_{\mathrm{s}}$ & 183 & 43 \\
\hline$T(K)$ & $3.9 \times 10^{3}$ & $5.1 \times 10^{4}$ \\
\hline$\theta, \Theta$ & 0.281 & 0.141 \\
\hline
\end{tabular}

In this Table, $R_{s}=\left(m_{H} e^{2} a / \hbar^{2}\right) \exp \left(-0.85 K_{s}\right)$, $\theta_{F}=2 m_{H} k_{B} T / \hbar^{2}\left(3 \pi^{2} n\right)^{2 / 3}, \mathrm{n}$ and $\mathrm{m}_{\mathrm{H}}$ refer to the number density and the mass of hydrogen (after [4]).

The data in Fig.2 elucidate physical mechanisms for the origin of strong magnetization in the magnetic WD [4]. For the nuclear FM to be of relevance to the stellar magnetism, the state of matter should fall in the FM domain. Solidification of metallic hydrogen, on the other hand, may not take place in such WD, since each of the $T_{\text {eff }}$ values exceeds the temperature at the triple point [4]. The FM phase transition is a concept which may provide a relevant account for an origin of the strong magnetization e.g. in degenerate magnetic stars.

\section{Analysis of experimental data}

\subsection{Metallic hydrogen}

The above work [5] was of great interest and excitation in the metallization of hydrogen at an unprecedentedly high pressure, reporting experimental Raman spectra, 
phase transition, metallization, and reflection coefficients observed visually. Unfortunately, the values of the electrical resistances obtained from their measured electrical resistances and the reduced dimensions of the sample was in order of magnitude larger than for the metal, and it was not clear to what extent hydrogen caused these observations [6].

In the beginning of this year, it appears an interesting paper [8] reporting the observation of the phenomenon of a phase transition, which indicates the production of metallic hydrogen. Atomic hydrogen from the liquid phase passed to a solid at a pressure up to $495 \mathrm{GPa}$ (see Fig.1) at a temperature of $5.5 \mathrm{~K}$ in a diamond anvil between the tips of artificial diamonds. Hydrogen from a state of transparent, like glass, transformed into a state of shiny metal, like copper or gold reflecting light (see Fig.3). The transition to the solid phase was confirmed by spectroscopic analysis. Then in [8] it was predicted that after removal of pressure, metallic hydrogen in the new experiments would remain metastable.

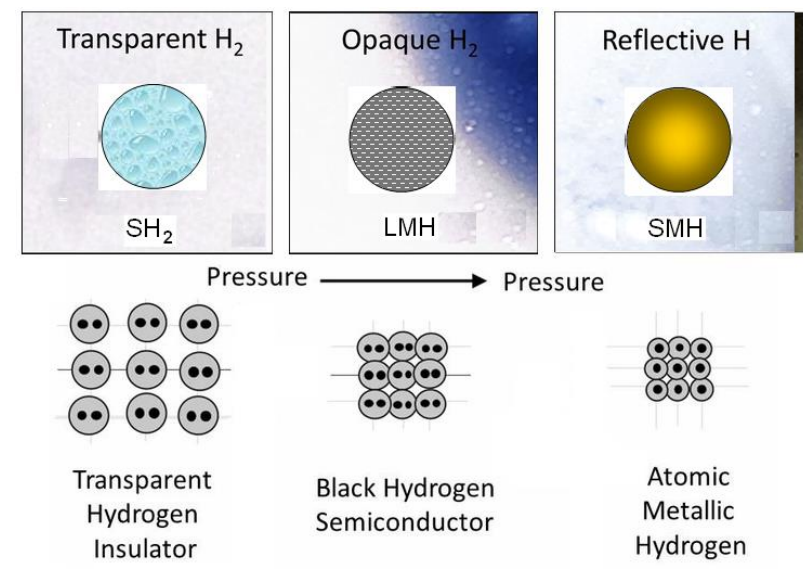

Fig. 3. Schematic illustration of reflectance in hydrogen [8].

However, a month later the first sample of metallic hydrogen was lost. Also in literature it was reported that now authors of [8] will be preparing another experiment to see if they can repeat high pressures in the first experiment and again make metallic hydrogen.

\subsection{Metallic sulfur hydrides}

As can be seen from the phase diagram for hydrogen (Fig.1), very high static pressures ( $500 \mathrm{GPa})$ are required to obtain a solid metallic phase. Conditions soften for a number of hydrides [7]. In [9], an attempt was made to realize these effects in a system with a hydrogen compound $\mathrm{H}_{2} \mathrm{~S}$, which is more stable. According to their measurements, the resistive curve in the normal state is linear in temperature, which is typical for metals, and at $\mathrm{T} \sim 200 \mathrm{~K}$ the electrical resistance begins to decrease - a resistive transition is observed. In their work, the temperature of the sharp drop of the electrical resistivity was identified with the onset of the transition to the SC state (Fig.4). However, the shape of the resistive curve with an inflection in the transition region indicated the possibility of an additional transition

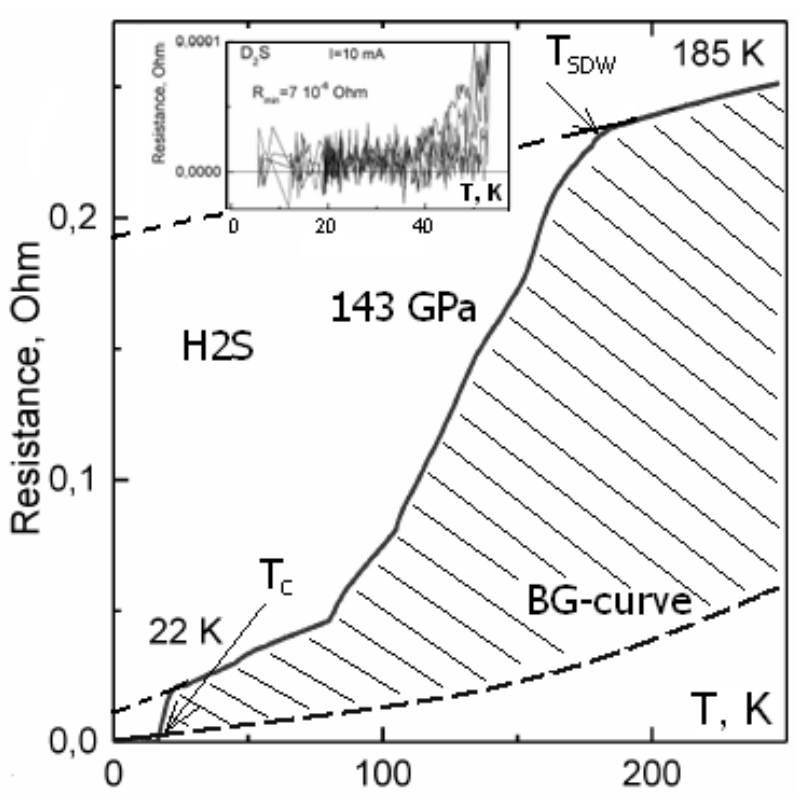

Fig. 4. T-dependence of resistance in $\mathrm{H}_{2} \mathrm{~S}$ (see $[9,10]$ ).

not directly related to SC. In our paper [10], it was suggested that the upper part of the resistive transition is associated with a magnetic (AF SDW-like) phase transition in the system (shaded area in Fig.4), while the $\mathrm{SC}$ transition occurs at much lower temperatures. The magnetic resistivity $\rho_{m}(T)$ can be estimated from the total resistivity $\rho_{\text {tot }}(T)$ via subtracting phonon contribution $\rho_{p h}(T)$ (Bloch-Gruneisen (BG) curve in Fig.4) and residual part $\rho_{\text {res }}$ (given in Fig.4 by the point of intersection of dashed lines with ordinate axis). This is reminiscent the picture observed in copper oxides and pnictides (selenides) of iron. While pure iron is known to be a FM, iron atoms in pnictides (selenides) are AFordered, what allows SC coexist with dynamical AFordering in the whole temperature (or pressure) range of interest (cf. [10,16]]). The same situation, apparently, is observed in metallic sulfur hydride. Moreover, for the solid-state phase of hydrogen, a layered structure is characteristic [1,7], as in the known HTSC compounds (see also [11]). In [11] the study of the evolution of the magnetic field within cavity in sulfur hydride at high pressures was performed as a function of temperature by means of synchrotron radiation (Fig.5), causing resonant nuclear scattering (NRS) in the system. As a magnetic field sensor, a strip of Sn foil was placed inside a cell with diamond anvil (DAC) filled with $\mathrm{H}_{2} \mathrm{~S}$. As it can be seen from Figs. 5 there is essential anisotropy in a NRS spectrum relative to the magnetic field direction what can be evidence of magnetic nature of the sample. The similar picture follows from measurements of magnetic field $H_{S n}(T)$ within the sulfur hydride sample (Fig.6). In accordance with [10] it can be proposed that upper part of drop of $\mathrm{H}_{\mathrm{Sn}}(\mathrm{T})$ curve can be due to drop of magnetic susceptibility $\chi_{A F}^{\prime \prime}$ characteristic for AF along AF-axis; SC drop of $H_{S n}(T)$ should occur only below. 


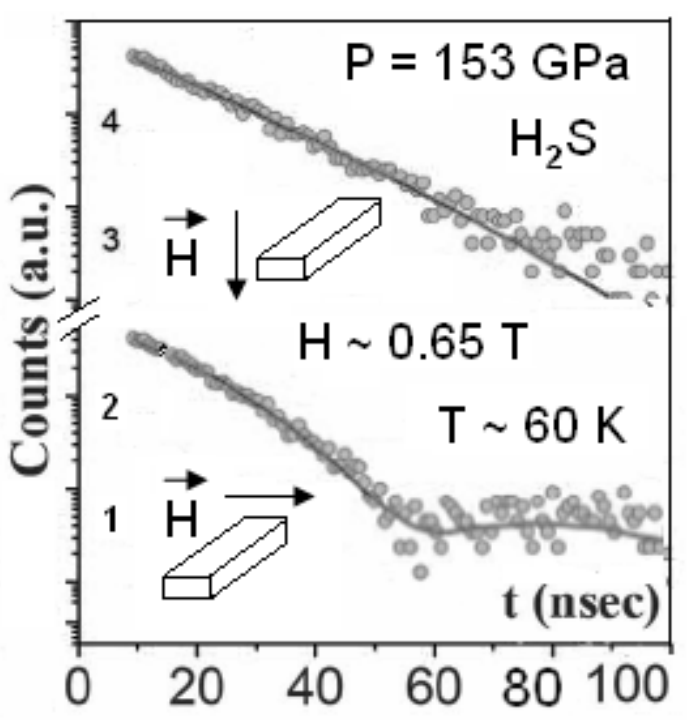

Fig. 5. Schematic anisotropy of NRS spectrum in $\mathrm{H}_{2} \mathrm{~S}$; in insert - geometry of experiment (the data are from [11]).

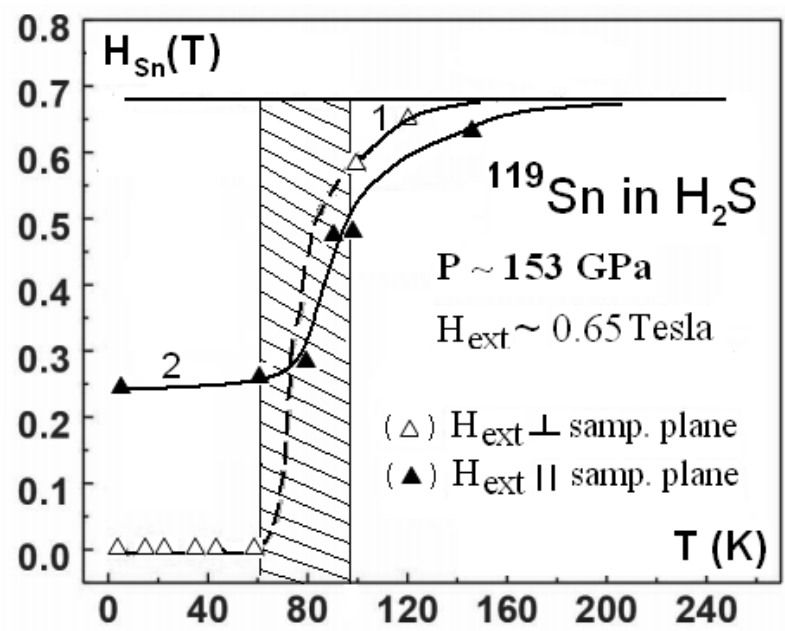

Fig. 6. Possible nonmonotonic disappearance (dashed curve [9]) of magnetic field in cavity within sulfur hydride; triangles are the experimental data from [11].

A detailed analysis of resonant nuclear scattering data performed in [12] demonstrates that the purely exponential nature of the spectrum corresponding to the SC state of the $\mathrm{H}_{2} \mathrm{~S}$ sample is observed only in the helium temperature range $\mathrm{T}<59 \mathrm{~K}$, and not up to $140 \mathrm{~K}$, as discussed in [11]. Since detailed data are not given in their work in the range $60 \mathrm{~K}<\mathrm{T}<100 \mathrm{~K}$ (shaded area in Fig.6), it can be assumed that the phase transition (dashed curve in Fig.6) has a complex (two-step character (cf. [9]) and its upper part can correspond to the magnetic (AF SDW-like) phase transition [10] and only its lower part relates to genuine SC transition.

\section{Discussion and conclusion}

So, the decrease in the resistance observed in $[9,11]$ at $\mathrm{T}$ $\sim 200 \mathrm{~K}$ can be related to the appearance in the hydrogen subsystem of a planar, modulated magnetic structure (AF SDW-like) (cf. [10,12]). The magnetic character of the transition, beginning at $\mathrm{T} \sim 200 \mathrm{~K}$, is indicated also by the anisotropy of NRS in orthogonal directions of the magnetic field [11]. As for drop in magnetization [9] at $200 \mathrm{~K}$, then "anomalous diamagnetism" was observed in $\mathrm{CuCl}$ [14] and in $\mathrm{YCaBaCuFeO}$ [15] before, however, $\mathrm{SC}$ was absent in these works.

This behavior is rather typical for systems with coexistence of dielectric and SC pairing (see, e.g. $[10,12])$. In such a system, with a decrease in $\mathrm{T}$, first a dielectric (AF SDW) transition takes place, and only then a SC transition occurs at lower temperatures. (The thermodynamics of dielectric (SDW) phase transition here is the same as for superconductor [13]). Such high $\mathrm{Tc}$ is a natural consequence of (e-e)-pairing at the background of high density of states which singularity arises in the narrow energy range near dielectric-(SDW)gap edges due to removing of electronic states from the energy region of dielectric (SDW) gap (already formed in the normal state). However, formation of the dielectric (SDW) gap at the Fermi surface leads to decreasing of energy region for (e-e)-pairing what, in its turn, leads, on the contrary, to decreasing of Tc.

Of course, to study the magnetic nature of normal-state dielectric phase transition in $\mathrm{H}_{2} \mathrm{~S}$ the additional efforts are necessary, and such more detailed search with "deploying a sensitive magnetic technique" is now in progress [17].

\section{References}

1. N.W.Ashcroft // Phys.Rev.Lett. V.21, 1748 (1968).

2. V.L.Ginzburg, V. V.Zheleznyakov, V.V. Zaitsev // Nature v. 220, 355 (1968).

3. J.E.Hirsch // Phys.Lett. A v.141, 191 (1989).

4. S.Ichimaru // Phys.Lett. A v. 258, 354 (1999).

5. M. I. Eremets \& I. A. Troyan // Nat.Mater. 10, 927 (2011).

6. W.J.Nellis et al. arXiv: cond-mat/1201.0407

7. N.W.Ashcroft // Phys.Rev.Lett. v.92, 187002 (2004).

8. R.P.Dias, I.F.Silvera // Science DOI: 10.1126 / science.aal1579 (2017).

9. A.P.Drozdov et al. // Nature v.525, 73 (2015).

10. L.S.Mazov // arXiv: cond-mat-1510.00123 (2015).

11. I.Troyan et al. // Science v.351, 1303 (2016).

12. L.S.Mazov // 14 conf. "Strongly correlated electronic systems and quantum critical phenomena "(HPPI RAS, Troitsk-Moscow, June, 2016), Conf.Abstr., P.17.

13. L.V. Keldysh, Yu.V. Kopaev // Fiz.Tv.Tela v.6, 2791 (1964).

14. N.B.Brandt et al. // JETP Lett. v.27, 37 (1978).

15. N.S.Ovanesyan et al. // Progress in HTSC, v.32 (World Scientific, Singapore, 1972), p.316.

16. Li Xiang et al. // Phys.Rev B 96, 024511 (2017).

17. C.W.Chu // High-Tc Copper Oxide Superconductors and Related Novel Materials (Eds. A.BussmannHolder et al., Springer Ser. in Mater. Sci. 255, Springer Int. Publ. AG 2017), p.15. 